\title{
Fostering a Sustainable Community in Batteries
}

As with nearly all facets of daily life, the COVID-19 pandemic has upended the traditional routines for science outreach and collaboration for battery researchers of all stripes. In-person conferences, meetings, lab visitations, and sabbaticals have largely been cancelled or postponed, disrupting the typical avenues for communication between scientists, engineers, and researchers. Increasingly, researchers have developed creative ways to leverage electronic communication formats, harnessing growing online social media communities to create ad-hoc replacements for the essential functions served by these conventional in-person events. Concurrently, there has been a growing recognition of the fundamental tension between travel-intensive scientific networking and the stated goals of many research fields focused on mitigating anthropogenic climate change and environmental degradation. Recent analysis of a European economics conference estimated roughly 0.5 tonnes of $\mathrm{CO}_{2}$ emissions per participant, while the University of California Santa Barbara recently estimated that conference travel accounts for roughly $30 \%$ of its carbon footprint. 112

Within this context, an online battery modeling community has taken shape. Centered around weekly webinars and a free-flowing Slack workspace, the community fulfills a critical need for connection between battery researchers with diverse backgrounds and interests

from all over the world. ${ }^{3}$ The community provides new avenues for information exchange, networking, and collaboration, which we hope will persist and provide a template for global, decentralized, democratic, and emissions-friendly community-building in a post-COVID science landscape. In this article, we describe the formation of this community, clearly state its mission, discuss initial activities, and identify challenges and opportunities, moving forward.

Origins of the community: Following a successful inaugural event in 2019, the 2020 
Oxford Battery Modeling Symposium (OBMS) ${ }^{4}$ was held on March 16 and 17, right at the start of COVID-related shutdowns in Europe and North America. About a week before the meeting on March 10, the organizing committee decided to go virtual. The virtual symposium used conferencing software (Zoom) in webinar mode for all presentations and Q\&A. Alongside, a messaging workspace (in Slack) was created for general discussion, which also provided an avenue for in-depth discussions amongst participants during the talks. Posters were uploaded to a messaging channel, and a subset of poster presenters were invited to present their work in the main presentation webinar. Most of the speakers also posted their slides in the messaging app. At the end of the symposium there was wide consensus that we had phenomenal momentum and the Slack community was gold. The text-driven messaging platform created a richer, more diverse dialogue with a flatter hierarchy than is typically supported at in-person conferences. Without the pressure of trying to fit discussions into crowded conference schedules, participants were able to pose and answer thought-provoking questions, respond with links to relevant citations, and carry the conversation forward over the course of days. However it was really the combination of webinar and messaging app that stood out as being more than the sum of its parts. Without the messaging app, the presentations would have been far less engaging, but without the presentations at specific dates/times, it would have been difficult to assemble everyone onto the messaging app. Having sufficient critical mass of engagement was pivotal in kick-starting the community.

Following this, we started the weekly Battery Modeling Webinar Series (BMWS). Online BMWS sessions have an open format, with frequent pauses for questions (on average one pause every 20 minutes), turning a presentation into a moderated organic discussion. At the time of writing this article, the webinar series had finished the first 11 webinars, with the next dozen already lined up. As described below, the webinar series covers a wide range of topics and has attracted a large, diverse, and highly engaged audience, leading to high impact for the early career researchers who have presented, so far.

The role of social media: The groundwork for a successful community was laid in part 
by the burgeoning battery community on Twitter, who typically share and discuss content under the \#battchat and \#batterytwitter hashtags. Even before the pandemic, there has been a growing recognition that social media and other electronic communication tools such as Twitter and Slack can supplement conference interactions in ways that are more flexible, more democratic, and less transient. ${ }^{5}$ Social media provides an additional avenue for early career researchers to network and establish identities that are unique from their advisors and mentors. This is especially important during the pandemic, which has sharply reduced the in-person networking opportunities that are critical for early career researchers. ${ }^{[6] 9}$ Moreover, Twitter bots such as @electrochemicat and @BatteryPapers, which automatically tweet relevant battery and energy storage papers, are a useful way to keep up with the rapidly growing literature. The battery community on Twitter has established norms centered on open-ended and collaborative dialogue, which helped create the necessary preconditions for a coherent and vibrant online community. The battery Twitter community also actively raised public awareness of and advertised OBMS and BMWS via tweets with the \#OBMS20 and \#BMWS hashtags, respectively, which were subsequently retweeted widely; a collage of OBMS and BMWS tweets is shown in Fig. 1. As a result, the BMWS community is diverse and hails from countries all over the world, as illustrated in Fig. 2 .

Vision and mission for the community: We see this as a persistent and inclusive community focused on battery modeling (broadly interpreted), with a formal weekly seminar series, an accompanying newsletter with around $\sim 700$ subscribers, and an informal communication platform on Slack. We think that this approach can provide great opportunities for industry engagement and input, as highlighted by community messages shown in Fig. 3.

The community and the seminar series have the following mission statements:

Community: Build an interdisciplinary global community of battery experts and enthusiasts to enable the free exchange of ideas, foster collaborations, and learn from one another.

Seminars: Create a platform for early career researchers (graduate students, postdoctoral scholars, junior faculty, early-career industry researchers and professionals) to discuss their 


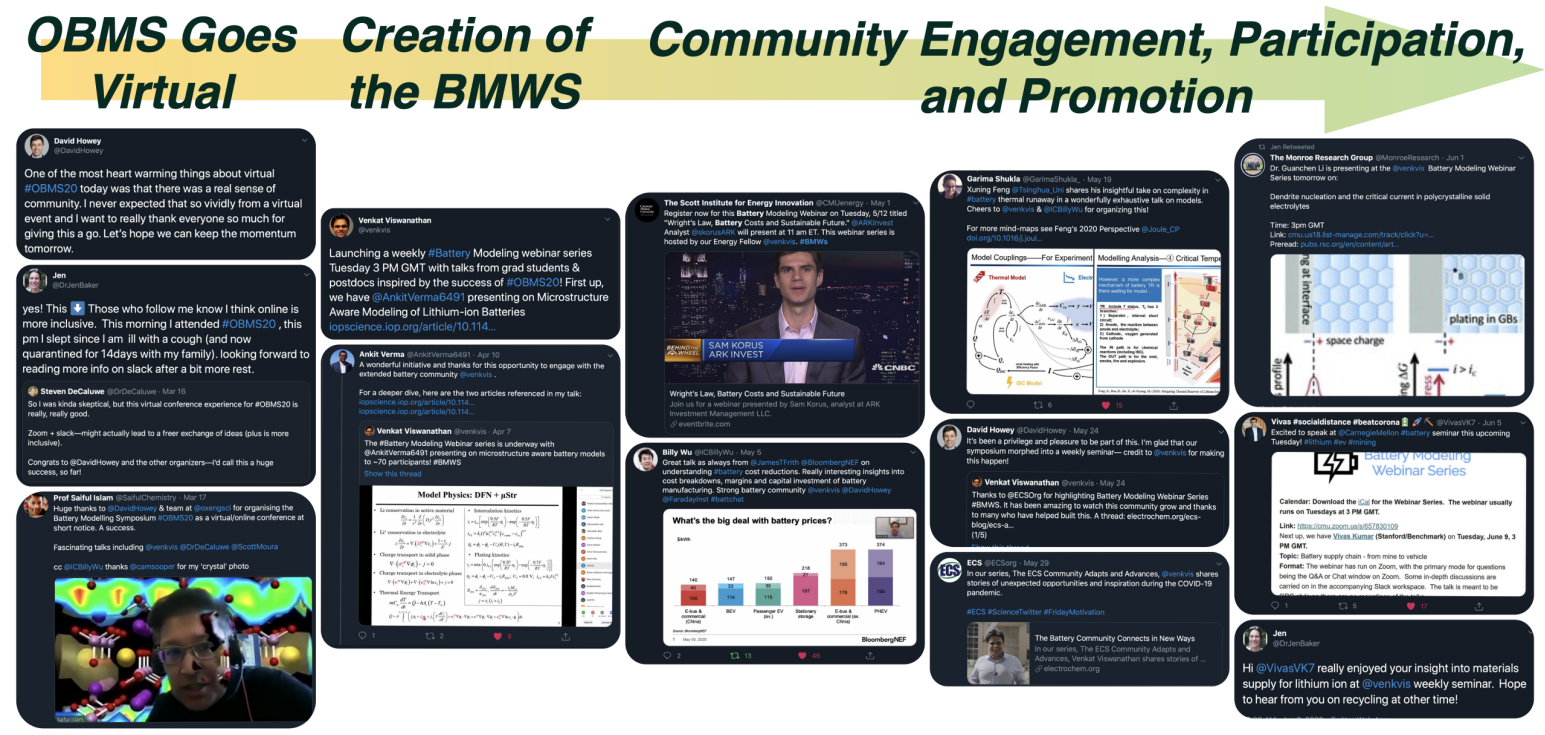

Figure 1: Social media has been pivotal in connecting the community and advertising and extending OBMS and BMWS virtual events.

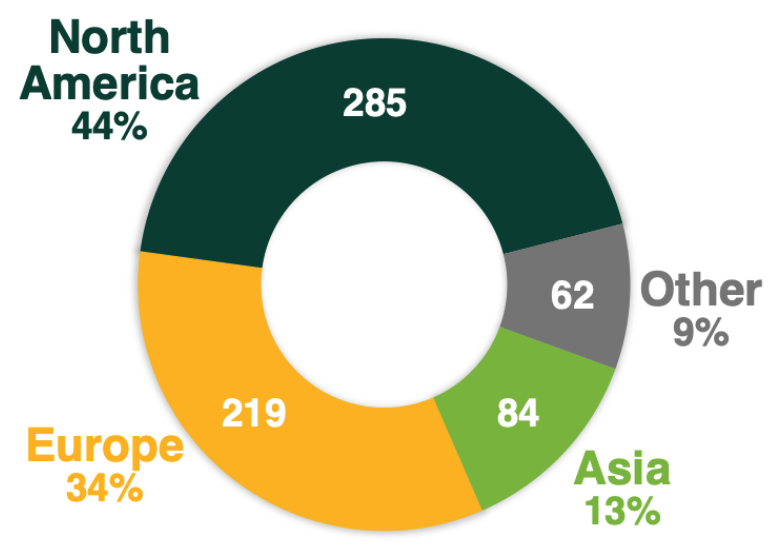

Figure 2: Locations reported by the 650 unique BMWS participants via Zoom through June 2020 . 
work with a interdisciplinary community of battery modeling experts, spanning from science to engineering and economics.

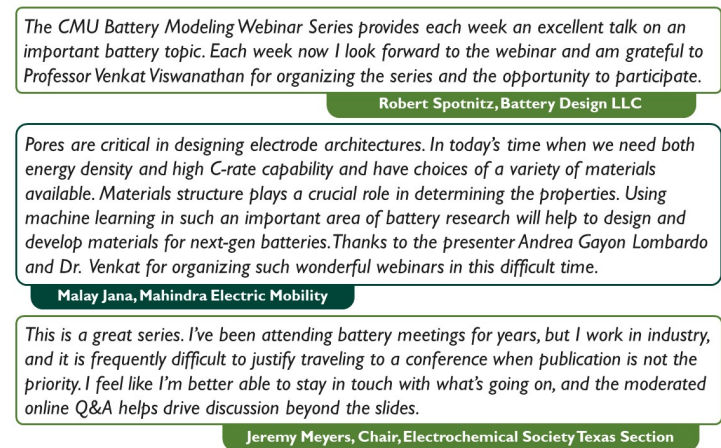

Figure 3: Community messages on the Webinar Series from industry researchers.

Wide range of battery modeling topics: The wide range of topics covered has helped broaden the knowledge-base of and form connections amongst the BMWS community, as indicated by community messages in Fig. 4. Here, we summarize the main insights from the talks so far (April-June 2020); an expanded version is given in the Supporting Information. The talks represent the wide range of focus and disciplines that fit under the banner of "battery modeling", as given in Table 1.

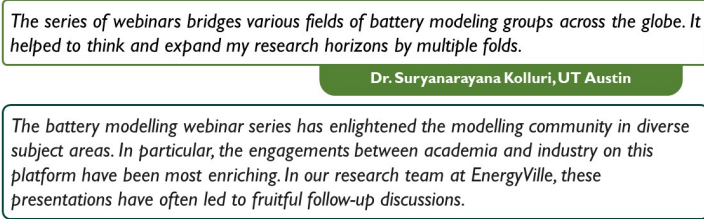

K. Chayambuka, VITO/EnergyVille, TU Eindhoven

Figure 4: Community messages on the wide range of topics covered.

Microstructure modeling: The first seminar was by Ankit Verma on the understanding the influence of microstructure on the thermal and degradation (Li plating) behavior of Li-ion batteries. $\frac{1011}{10}$ Ankit showed that there is significant difference between heat generation using the commonly-employed Bruggeman approximation, as opposed to those obtained using the microstructures directly. Ankit also demonstrated how detailed microstructure analysis becomes important for fast charging applications. Andrea Gayon Lombardo presented on 
generative adversarial networks to digitally generate realistic electrode microstructures. 12 Such deep learning methods can generate a range of electrode microstructures with less cost and effort than physical fabrication methods. These microstructures can then be used as input into battery simulations to guide development of new, high-performance electrodes.

Data-driven methods and modeling: Data-driven methods can accurately and efficiently predict charge-discharge performance and battery lifetime. Peter Attia demonstrated a machine learning (ML) approach to optimize a parameter space which dictates the current and voltage profiles during fast charging.13 The approach utilizes Bayesian optimization ${ }^{14}$ to identify fast charge protocols by sampling the design space efficiently. These methods have seen increasing use in searching design spaces of electrochemical systems. $\frac{15 \mid 16}{16}$ Yunwei Zhang presented an approach to use Gaussian process regression on over 20,000 electrochemical impedance spectra (EIS) from cells with varying degrees of capacity degradation. ${ }^{17}$ Gaussian processes have become popular recently for modelling battery health. ${ }^{18 \mid 19}$ Last, Jiapeng Liu delivered a talk on metrics to assess EIS data quality. In their work, they have developed a Bayesian Hilbert transform to interpret impedance data. 20 Jiapeng also discussed metrics to score compliance of EIS data to Kramers-Kronig relation, and supplementing conventional metrics based on residuals and estimated means with information-theoretic concepts. $\underline{20}$

Battery cost and resource modeling: Understanding current and future battery cost is crucial for a range of stakeholders. James Frith, Sam Korus and Vivas Kumar delivered thought-provoking talks on bottom-up and top-down approaches to cost modelling and supply chain considerations. As discussed further in the Supporting Information, Sam discussed Wright's Law, $\stackrel{21}{23}$ which shows that historically, lithium-ion battery cost (USD/kWh) decreases by about 18 percent for every doubling in cumulative installed capacity. Using similar methods, James forecasts lithium-ion battery pack cost to reach $61 \mathrm{USD} / \mathrm{kWh}$ by 2030, compared to current costs of 150 USD/kWh. 24 James introduced the BNEF bottomup, component-based cost model "BattMan", which highlights the importance of managing battery material costs, most notably the cathode. ${ }^{25}$ Approaches to lowering battery cost 
were discussed, including managing material costs, increasing battery energy density, yield, and utilization of manufacturing facilities. Finally, Vivas provided a deep dive into battery supply chain, $\sqrt{26}$ and pointed out the absence of commoditization in batteries, which enables differentiation of cathode chemistries for different applications and customers. ${ }^{27}$

Physics-based modeling: Xuning Feng discussed battery safety and electrochemical-thermal Li-ion battery models. $\stackrel{28}{2}$ The talk discussed (i) rapid capacity degradation at high temperature, (ii) internal short circuits due to separator failure, and (iii) thermal runaway caused by heat release at extreme temperatures. Jorn Reniers reviewed an exhaustive list of existing models on capacity fade and battery degradation and compared them, demonstrating the extent to which the predictions from these models vary relative one another and to experimental data. ${ }^{29}$ These comparisons were carried out with an open-source codebase developed along with the related publication. ${ }^{30}$ Guanchen Li presented work on electro-chemo-mechanical coupling to explain dendrite nucleation at solid electrolyte interfaces. 31

Recent Outcomes: It will take time to see the long-term impact of BMWS. In the near-term, it has undoubtedly provided professional growth and enhanced visibility for early career researchers. There has been a significant upsurge in article reads/downloads for the works presented, with data accessed from IOP publishing ${ }^{32}$ listed in Table 2 . Refs. 10 and 11 had an increase in downloads from 70 and 152 to 1549 and 2060, respectively. Ref. [10 was recently featured in the Electrochemical Society April newsletter as one of the top downloaded articles. ${ }^{33}$ Subsequent talks covering J. Electrochem. Soc. articles have also witnessed excellent growth in downloads, compared to at the time of seminar announcement; 156 to 501 for Feng et al. ${ }^{28}$ and 633 to 1162 for Reniers et al. ${ }^{29}$ In addition, one of the speakers (Verma) saw a 5000\% rise in professional network views (LinkedIn) during the presentation week. Alongside this, the webinar series provides professional development to researchers new to the battery research area, as shown in the community messages in Fig. 5

Concurrent Developments: Beyond modeling, the wider battery and electrochemistry community has adopted virtual channels for knowledge dissemination. Energy storage webi- 
Table 1: Summary of the Battery Modeling Webinar Series (BMWS).

\begin{tabular}{|c|c|c|c|}
\hline Date & Speaker & Title & Reads \\
\hline April 7, 2020 & Ankit Verma & $\begin{array}{l}\text { Microstructure Aware } \\
\text { Lithium-ion Batteries }\end{array}$ & Refs. 10, 11 \\
\hline April 14, 2020 & Peter Attia & $\begin{array}{l}\text { Closed-loop optimization of battery perfor- } \\
\text { mance with machine learning }\end{array}$ & Ref. 13 \\
\hline April 21, 2020 & Yunwei Zhang & $\begin{array}{l}\text { Identifying degradation patterns of batter- } \\
\text { ies from impedance using ML }\end{array}$ & Ref. 17 \\
\hline April 28, 2020 & Jiapeng Liu & $\begin{array}{l}\text { Bayesian Hilbert transform (BHT) method } \\
\text { for assessing impedance data }\end{array}$ & Ref. 20] \\
\hline May 5, 2020 & James Frith & $\begin{array}{l}\text { How BattMan Can Help Us to Understand } \\
\text { Battery Cost Declines }\end{array}$ & Ref. 24 \\
\hline May 12,2020 & Sam Korus & Top-down modeling of battery cost & Ref. 22 \\
\hline May 19,2020 & Xuning Feng & $\begin{array}{l}\text { Electrochemical-Thermal Coupled Battery } \\
\text { Thermal Runaway Model }\end{array}$ & Ref. 28 \\
\hline May 26, 2020 & Jorn Reniers & $\begin{array}{l}\text { Review of physics-based continuum bat- } \\
\text { tery degradation models }\end{array}$ & Refs. 29, 30 \\
\hline June 2, 2020 & Guanchen Li & $\begin{array}{l}\text { Dendrite Nucleation and Critical Currents } \\
\text { in Polycrystalline Solid Electrolytes }\end{array}$ & Ref. 31 \\
\hline June 9, 2020 & Vivas Kumar & Battery supply chain - from mine to vehicle & Ref. 26 \\
\hline June 16,2020 & $\begin{array}{l}\text { Andrea } \\
\text { Gayon Lom- } \\
\text { bardo }\end{array}$ & $\begin{array}{l}\text { Pores for thought: Design and optimisa- } \\
\text { tion of electrode microstructure using ma- } \\
\text { chine learning }\end{array}$ & Ref. 12 \\
\hline
\end{tabular}

nars have exploded in quantity, with 38 free-to-attend talks held in June 2020 alone. ${ }^{34}$ While BMWS showcases early career researchers, Stanford's StorageX symposia have invited titans of the field, providing global access to plenary-level lectures once reserved for exclusive (and expensive) conferences. ${ }^{35}$ The San Francisco Bay Area's monthly "Battery Brunch", 36 originally an informal gathering of local battery industry professionals, investors, policy makers, and academics, has more than doubled its membership (from 223 to 557) since updating

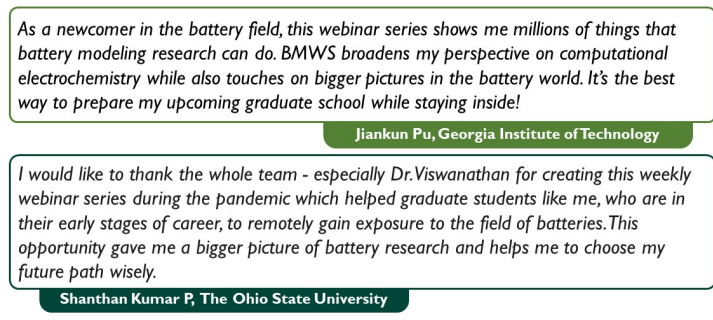

Figure 5: Community messages on professional development for early-career researchers. 
to a virtual format in April 2020. Members of the BMWS community Andrew Wang and Nicholas Yiu have published an email newsletter called Intercalation Station. ${ }^{37}$ The monthly newsletter covers battery technology developments in research and industry and highlights related lively dialogues from Twitter. YouTube is a growing platform for battery professionals, academics, and enthusiasts to share content, such as The Limiting Factor channel on battery patent reviews. $\stackrel{38}{3}$ Webinar recordings have an added benefit of catering to asynchronous schedules. Undoubtedly, research communities across the energy storage domain are arriving at a new paradigm for sharing and discussing scientific progress.

Table 2: Article Download Metrics: Before (accessed on seminar announcement date) and After (accessed on June 15, 2020)

\begin{tabular}{|c|c|c|}
\hline Articles & Prior Downloads & Current Downloads \\
\hline Ref. $[10$ & 70 & 1549 \\
\hline Ref. $[11$ & 152 & 2060 \\
\hline Ref. $[28$ & 156 & 501 \\
\hline Ref. $[29$ & 633 & 1162 \\
\hline
\end{tabular}

Sustainability and Inclusiveness: An important consideration is the carbon emissions mitigated by online conferencing. Battery researchers must be cognizant of the climate emergency, as international air travel dominates the carbon budgets of academics. ${ }^{39}$ The BMWS community has welcomed ca. 650 unique attendees spanning 6 continents virtually, as shown in Fig. 2, To a first approximation, assuming attendees travel from their geographically-local major hub to a North American location, flights to and from an in-person BMWS conference would contribute approximately 700 tonnes of carbon dioxide equivalent $\left(\mathrm{CO}_{2} \mathrm{e}\right) .40141$ Even applying a discount of $50 \%$ to account for lower in-person attendance, 350 tonnes $\mathrm{CO}_{2} \mathrm{e}$ is comparable to the annual electricity consumption of 59 US households. Offsetting these emissions would require ca. 450 acres of US forest growth, annually. Putting into context of an individual researcher, flying from the UK to the US adds 1.2 tonnes to the 5.2 tonne carbon footprint of the average UK researcher. .42

Conferences are places to cross-pollinate ideas and form friendships as well as collaborations. Although one forgoes the spontaneity and depth of face-to-face interactions, virtual 
networking has other accessibility advantages. More-so than traditional settings, the flat hierarchy of virtual dialogue enables interaction between senior and junior researchers, and open dialogues lower the activation barrier for a more diverse set of BMWS members to contribute. Many of the exclusionary limitations such as funding, travel constraints, and caring responsibilities are also alleviated. Participants can choose to modulate their engagement based on availability. Most researchers have become adept with virtual social and collaborative tools in recent months. Going forward, this format serves as a valuable supplement to in-person meetings. However, this will require attendees to adapt to and stick with these new technologies for online engagement to be effective.

Outlook: Challenges and Opportunities A battery is a complex, developing system, and there are countless opportunities for a thriving and emerging online community. This includes but is not limited to expanded education for all participants, new collaborations, diverse engagement, generation of research ideas, and multi-disciplinary projects. There will be many challenges to adopt and grow a new medium, including finding a common dialogue and locus of expertise, overcoming language barriers, and maintaining high quality content. An important development area is bridging the collaboration gap between the largest industry players and the academic community in energy storage. Korea, China, and Japan host four of the top five manufacturers of lithium-ion batteries. ${ }^{26}$ Inclusion of these groups will unlock great opportunities to deploy scientific advancements on larger scales.

An ambitious goal for this community is to perform global, massively collaborative, cutting-edge research in batteries, along the lines of the Polymath project. $\stackrel{43}{ }$ There are numerous cross-disciplinary challenges in batteries and electric mobility. $\frac{44}{}$ Owing to the diverse backgrounds of our members, we could bring together a very unique approach to critical problems in battery science and technology. Additional open-source software and scientific tools ${ }^{45[46}$ such as PyBaMM (Python Battery Mathematical Modelling) software, ${ }^{47}$ MPET (Multiphase Porous Electrode Theory) software, $\stackrel{48}{4}$ and Cantera, ${ }^{49}$ will be needed to achieve this goal. Nonetheless, this article itself serves as an important proof point that distributed 
self-assembled collaborations are possible. We view the entire community as one cohesive collaborative research enterprise, along the lines of the community message in Fig. 6

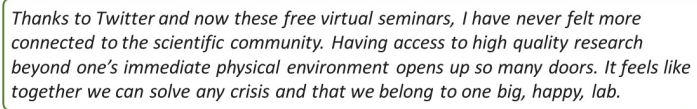

Figure 6: Community message on viewing the group as one big, happy lab!

As scientists, it is our duty to be at the forefront of positive innovation. We promote sustainable, positive behaviour change by interacting with one another, by suggesting an effective way to reduce environmentally deleterious networking. We see intense, emissionsheavy travel schedules as a thing of the past. ${ }^{50}$ Furthermore, as scientists, we have to keep dreaming: maybe, one day, networking and conferences will happen with only minimal barriers, and become even more inclusive in virtual reality rooms.

Resources: We list below links for the resources talked about in the article:

Battery Modeling Webinar Series: Webinar Link:

https://cmu.zoom.us/s/657830109 (typically 3 pm GMT on Tuesdays).

Battery Modeling Webinar Series Newsletter: Subscribe at

http: //andrew.cmu.edu/user/venkatv/bmws.html

Intercalation Station Monthly Newsletter. Subscribe at

http://intercalationstation.substack.com/

\section{Acknowledgement}

The team acknowledge support for Zoom license and promotion from Carnegie Mellon University, Oxford University and Scott Institute for Energy Innovation at Carnegie Mellon. UU would like to acknowledge Dr. Rinaldo Raccichini and Teresa Gallo for the fruitful discussion on environmental issues. DAH thanks the organisers of OBMS including Jon Chapman, 
Charles Monroe, Colin Please and the events team in Engineering Science at Oxford University.

\section{AUTHOR INFORMATION}

Jenny A. Baker (College of Engineering, Swansea University, United Kingdom) Twitter: @DrJenBaker

Martin Beuse (ETH Zurich, Zurich, Switzerland) Twitter: @mart_di_be

Steven C. DeCaluwe (Department of Mechanical Engineering, Colorado School of Mines, Golden, CO, United States) Twitter: @DrDeCaluwe

Linda W. Jing (Independent Researcher, United States) Twitter: @LindaWJing

Edwin Khoo (Institute for Infocomm Research, Agency for Science, Technology and Research, Singapore) Twitter: (edwinksl

Shashank Sripad (Department of Mechanical Engineering, Carnegie Mellon University, Pittsburgh, PA, United States) Twitter: @ssripad1

Ulderico Ulissi (Independent Researcher, United Kingdom) Twitter: @UldericoUlissi Ankit Verma (National Renewable Energy Laboratory, Golden, CO, United States) Twitter: @AnkitVerma6491

Andrew A. Wang (Department of Engineering Science, University of Oxford, United Kingdom) Twitter: @ndrewwang

Yen T. Yeh (Voltaiq Inc., Berkeley, CA, United States) Twitter: @YenYeh

Nicholas Yiu (Independent Researcher, United Kingdom) Twitter: @NicholasYiu

David A. Howey (Department of Engineering Science, University of Oxford, and Faraday Institution) Twitter: @DavidHowey

Venkatasubramanian Viswanathan (Department of Mechanical Engineering, Carnegie Mellon University, Pittsburgh, PA, United States) Twitter: @venkvis

\section{Corresponding Author}

*E-mail: venkvis@cmu.edu 


\section{Notes}

Views expressed in this Energy Focus are those of the authors and not necessarily the views of the ACS. The authors declare no competing financial interest.

\section{References}

(1) Desiere, S. The Carbon Footprint of Academic Conferences: Evidence from the 14th EAAE Congress in Slovenia. EuroChoices 2016, 56-61.

(2) Hiltner, K. A Nearly Carbon-Neutral Conference Model. 2020; https://hiltner. english.ucsb.edu/index.php/ncnc-guide/, Accessed: 11-June-2020.

(3) Jin, S. COVID-19, Climate Change, and Renewable Energy Research: We Are All in This Together, and the Time to Act Is Now. ACS Energy Lett. 2020, 5, 1709-1711.

(4) Howey, D.; Monroe, C.; Please, C. Oxford Battery Modeling Symposium. 2020; https: //batterymodel.ox.ac.uk/, Accessed: 12-June-2020.

(5) Khoo, E.; Lacey, M. J.; DeCaluwe, S. C. Social Media Platforms for Electrochemistry. Electrochem. Soc. Interface 2019, 28, 41.

(6) Avasthi, P.; Neill, U. S.; Porter, K. M. P.; Weeraratna, A. T. Preserve junior faculty in biomedical sciences during and after the pandemic. Nat. Med. 2020, 1-2.

(7) Heemstra, J. M. A Scientist's Guide to Social Media. ACS Cent. Sci. 2020, 6, 1-5.

(8) Dauenhauer, P. J. Expand Your Academic Impact with Social Media Best Practices. Matter 2020, 2, 789-793.

(9) Levine, R. L.; Rathmell, W. K. COVID-19 impact on early career investigators: a call for action. Nat. Rev. Cancer 2020, 1-2. 
(10) Chen, C.-F.; Verma, A.; Mukherjee, P. P. Probing the role of electrode microstructure in the lithium-ion battery thermal behavior. J. Electrochem. Soc. 2017, 164, E3146E3158.

(11) Vishnugopi, B. S.; Verma, A.; Mukherjee, P. P. Fast Charging of Lithium-ion Batteries via Electrode Engineering. J. Electrochem. Soc. 2020, 167, 090508.

(12) Gayon-Lombardo, A.; Mosser, L.; Brandon, N. P.; Cooper, S. J. Stochastic reconstruction of periodic, three-dimensional multi-phase electrode microstructures using generative adversarial networks. arXiv preprint arXiv:2003.11632 2020,

(13) Attia, P. M. et al. Closed-loop optimization of fast-charging protocols for batteries with machine learning. Nature 2020, 578, 397-402.

(14) Grover, A.; Markov, T.; Attia, P.; Jin, N.; Perkins, N.; Cheong, B.; Chen, M.; Yang, Z.; Harris, S.; Chueh, W., et al. Best arm identification in multi-armed bandits with delayed feedback. arXiv preprint arXiv:1803.10937 2018,

(15) Dave, A.; Mitchell, J.; Kandasamy, K.; Burke, S.; Paria, B.; Poczos, B.; Whitacre, J.; Viswanathan, V. Autonomous discovery of battery electrolytes with robotic experimentation and machine-learning. arXiv 2019, 2001.09938 [physics.app-ph].

(16) Nakano, K.; Noda, Y.; Tanibata, N.; Takeda, H.; Nakayama, M.; Kobayashi, R.; Takeuchi, I. Exhaustive and informatics-aided search for fast Li-ion conductor with NASICON-type structure using material simulation and Bayesian optimization. APL Materials 2020, 8, 041112 .

(17) Zhang, Y.; Tang, Q.; Zhang, Y.; Wang, J.; Stimming, U.; Lee, A. A. Identifying degradation patterns of lithium ion batteries from impedance spectroscopy using machine learning. Nat. Comm. 2020, 11, 1-6. 
(18) Richardson, R. R.; Osborne, M. A.; Howey, D. A. Gaussian process regression for forecasting battery state of health. J. Power Sources 2017, 357, 209-219.

(19) Lucu, M.; Martinez-Laserna, E.; Gandiaga, I.; Liu, K.; Camblong, H.; Widanage, W.; Marco, J. Data-driven nonparametric Li-ion battery ageing model aiming at learning from real operation data-Part B: Cycling operation. Journal of Energy Storage 2020, 30, 101410 .

(20) Liu, J.; Wan, T. H.; Ciucci, F. A Bayesian View on the Hilbert Transform and the Kramers-Kronig Transform of Electrochemical Impedance Data: Probabilistic Estimates and Quality Scores. ChemRxiv 2020, DOI: 10.26434/chemrxiv.12152529.

(21) Wright, T. P. Factors affecting the cost of airplanes. J. Aero. Sci. 1936, 3, 122-128.

(22) Nagy, B.; Farmer, J. D.; Bui, Q. M.; Trancik, J. E. Statistical basis for predicting technological progress. PLoS One 2013, 8.

(23) Schmidt, O.; Hawkes, A.; Gambhir, A.; Staffell, I. The future cost of electrical energy storage based on experience rates. Nat. Energy 2017, 2, 1-8.

(24) Electric Vehicle Outlook 2020. 2020; https://about.bnef.com/ electric-vehicle-outlook/, Accessed: 11-June-2020.

(25) Kwade, A.; Haselrieder, W.; Leithoff, R.; Modlinger, A.; Dietrich, F.; Droeder, K. Current status and challenges for automotive battery production technologies. Nat. Energy 2018, 3, 290-300.

(26) Moores, S.; Rawles, C.; Miller, A. Who is Winning The Global Lithium Ion Battery Arms Race? 2019; https://www.benchmarkminerals.com/ who-is-winning-the-global-lithium-ion-battery-arms-race/, Accessed: 11-June-2020. 
(27) Beuse, M.; Schmidt, T. S.; Wood, V. A "technology-smart" battery policy strategy for Europe. Science 2018, 361, 1075-1077.

(28) Feng, X.; He, X.; Ouyang, M.; Wang, L.; Lu, L.; Ren, D.; Santhanagopalan, S. A coupled electrochemical-thermal failure model for predicting the thermal runaway behavior of lithium-ion batteries. J. Electrochem. Soc. 2018, 165, A3748-A3765.

(29) Reniers, J. M.; Mulder, G.; Howey, D. A. Review and performance comparison of mechanical-chemical degradation models for lithium-ion batteries. J. Electrochem. Soc. 2019, 166, A3189-A3200.

(30) Reniers, J. M.; Howey, D. A. Slide (simulator for lithium-ion degradation). https: //github.com/davidhowey/SLIDE, 2019; Accessed: 10-June-2020.

(31) Li, G.; Monroe, C. W. Dendrite nucleation in lithium-conductive ceramics. Phys. Chem. Chem. Phys. 2019, 21, 20354-20359.

(32) IOP Publishing. 2020; https://ioppublishing.org/, Accessed: 18-June-2020.

(33) Staff, E. S. Electrochemical Society Weekly News - May 26th 2020. 2020; https://es.informz.net/informzdataservice/onlineversion/ind/ bWFpbGluZ2luc3RhbmN1aWQ90TM10DA0MSZzdWJzY3JpYmVyaWQ9MTEwMzAxNjU3MQ==,

Accessed: 12-June-2020.

(34) Wang, A. A. Public Calendar: Battery \& Energy Storage Webinars. 2020; https: //calendar.google.com/calendar/embed?src=kn0ioj0nghabtevt9er438g5740 group.calendar.google.com\&ctz=Europe/London\&pli=1, Accessed: 10-June-2020.

(35) Golden, M. Lithium batteries dominate, but need to improve a lot, researchers agree at StorageX Symposium. 2020; Accessed: 10-June-2020.

(36) Yeh, Y.; Jing, L. Battery Brunch. 2020; https://www.facebook.com/groups/ 395246738065263/, Accessed: 14-June-2020. 
(37) Wang, A. A.; Yiu, N. Intercalation Station. 2020; https://intercalationstation. substack.com/p/-intercalation-station-issue-2, Accessed: 10-June-2020.

(38) Giesige, J. Tabless Electrode Patent (Deep Dive), The Limiting Factor. 2020; https: //youtu.be/Rcw3vA0gAMs, Accessed: 11-June-2020.

(39) Editorial: Rethinking conferences. Nat. Rev. Phys. 2020, 2, 67.

(40) Carbon Footprint Ltd., Carbon Footprint Calculator. 2020; https://www. carbonfootprint.com/calculator.aspx, Accessed: 11-June-2020.

(41) Ulissi, U. BMWS - Considerations around sustainability. Figshare 2020, DOI: 10.6084/m9.figshare.12473396.

(42) UK Provisional Greenhouse Gas Emissions. 2020; https://assets.publishing. service.gov.uk/government/uploads/system/uploads/attachment_data/ file/875482/2019_UK_greenhouse_gas_emissions_provisional_figures_ statistical_summary.pdf, Accessed: 13-June-2020.

(43) Gowers, T.; Nielsen, M. Massively collaborative mathematics. Nature 2009, 461, 879881.

(44) Crabtree, G. The coming electric vehicle transformation. Science 2019, 366, 422-424.

(45) Howey, D. A. Tools for Battery Health Diagnostics and Prediction. Electrochem. Soc. Interface 2019, 28, 55-56.

(46) DeCaluwe, S. C. Open Software for Chemical and Electrochemical Modeling: Opportunities and Challenges. Electrochem. Soc. Interface 2019, 28, 47-50.

(47) Sulzer, V.; Marquis, S. G.; Timms, R.; Robinson, M.; Chapman, S. J. Python Battery Mathematical Modelling (PyBaMM). ECSarXiv. February 2020, 7, DOI: 10.1149/osf.io/67ckj. 
(48) Smith, R. B.; Bazant, M. Z. Multiphase Porous Electrode Theory. J. Electrochem. Soc. 2017, 164, E3291-E3310.

(49) Goodwin, D. G.; Speth, R. L.; Moffat, H. K.; Weber, B. W. Cantera: An Objectoriented Software Toolkit for Chemical Kinetics, Thermodynamics, and Transport Processes. https://www . cantera.org, 2018; Version 2.4.0.

(50) Wynes, S.; Nicholas, K. A. The climate mitigation gap: education and government recommendations miss the most effective individual actions. Environ. Res. Lett. 2017, 12, 074024 . 